\title{
ESTRATÉGIAS TERAPEUTICAS OCUPACIONAIS ALIADAS À TECNOLOGIA ASSISTIVA FAVORECENDO A EXECUÇÃO DAS ATIVIDADES DA VIDA DIÁRIA DE PACIENTES ASSISTIDOS PELO SERVIÇO DE ASSISTÊNCIA DOMICILIAR DO HOSPITAL GERAL DR. WALDEMAR ALCÂNTARA.
}

\author{
OLIVEIRA, Ítala de Brito (1); \\ GOES, Leirylane de Souza Pereira (2); \\ (1) Hospital Geral Dr. Waldemar Alcântara, Terapeuta Ocupacional, Especialista em \\ Saúde Integral da Criança e do adolescente na Estratégia de Saúde da Família. \\ e-mail:italaolyveira@gmail.com \\ (2) Hospital Geral Dr. Waldemar Alcântara, Enfermeira, Mestre em Saúde Pública. \\ e-mail:leiryufc@gmail.com
}

\section{RESUMO (11 PTS, NEGRITO)}

\begin{abstract}
As Atividades de Vida Diária são componente importante no papel de manutenção pessoal, quando há uma disfunção ocupacional, a interação social do indivíduo é alterada e o terapeuta ocupacional é o profissional especialista na readequação da rotina. Estratégias são necessárias para o resgate da execução destas atividades, e neste estudo aliamos a terapia ocupacional à tecnologia assistiva e alcançamos resultados positivos no que tange a funcionalidade humana, mesmo não alcançando a independência total conforme os escores da Medida de Independência Funcional, proporcionamos aos indivíduos aqui citados, o seu protagonismo, mesmo estando em Assistência Domiciliar portando doenças crônicas degenerativas.
\end{abstract}

Palavras-chave: Terapia Ocupacional; Tecnologia Assistiva; Atividades da Vida Diária.

\begin{abstract}
PTS, NEGRITO)
The everyday activities are important elements in the role of self maintainability. Once there is an ocupational disfunction, the Ocupational Therapeut is the professional specialist skilled to readjust this routine. As strategies are necessary to hold back these routine activities performances, we put together Ocupational Therapy and Assistive Technology and reached positive results related to human functionality. Even though, according to the Functional Independance Measurement scores, we haven't reached the total independance, we gave protagonism to the individuals mentioned here who were under Home Assistance due to chronic degenerative deseases.
\end{abstract}

Keywords: Ocupacional Therapy; Assistive Technology; Everyday activities. 


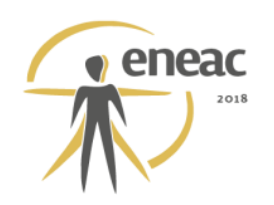

\section{INTRODUÇÃO}

Com foco na assistência humanizada e a garantia de continuidade dos cuidados à saúde, o Serviço de Assistência Domiciliar - SAD possibilita a desinstitucionalização de pacientes que se encontram internados nos serviços hospitalares, além de evitar hospitalizações desnecessárias de acordo com os princípios do Sistema Único de Saúde.

Para garantir um Serviço de Assistência Domiciliar é necessário uma equipe multidisciplinar especializada, geralmente, composta por médico, enfermeiro, assistente social, fisioterapeuta, fonoaudiólogo, dentista, farmacêutico, nutricionista, psicólogo e terapeuta ocupacional, que trabalhe de forma alinhada proporcionando uma assistência integral, segura e humanizada.

Existem separações nítidas entre as ocupações, porém uma equipe que funciona bem é aquela em que a passagem do paciente de uma modalidade terapêutica para outra é harmoniosa (EGGERS, 1984).

Na Atenção Domiciliar (AD), o acolhimento como postura da equipe faz mais sentido, afinal, trata-se de um âmbito que percebe a dor e o sofrimento em todas as suas facetas, com o conhecimento do espaço de vida do usuário. (BRASIL 2013). Podemos conhecer não só o paciente, mas a realidade de seu cotidiano, bem como de seus familiares, proporcionando assim um melhor vínculo não só com o paciente, mas também com os cuidadores envolvidos.

A terapia ocupacional preconiza o envolvimento de familiares ao tratamento tendo como consequência um maior entendimento da essência da incapacidade. Lucrando o paciente e cuidadores, resultando no alcance dos objetivos terapêuticos traçados (EGGERS, 1984).

O Conselho Federal de Fisioterapia e Terapia Ocupacional (COFFITO, 2018), define a Terapia Ocupacional como a profissão de nível superior voltada aos estudos, à prevenção e ao tratamento de indivíduos portadores de alterações cognitivas, afetivas, perceptivas e psicomotoras, decorrentes ou não de distúrbios genéticos, traumáticos e/ou de doenças adquiridas, através da sistematização e utilização da atividade humana como base de desenvolvimento de projetos terapêuticos específicos, na atenção básica, média complexidade e alta complexidade.

O referido órgão refere também que o Terapeuta Ocupacional é o profissional que compreende a Atividade Humana como um processo criativo, criador, lúdico, expressivo, evolutivo, produtivo e de auto manutenção e o Homem, como um ser práxico interferindo no cotidiano do usuário comprometido em suas funções práxicas objetivando alcançar uma melhor qualidade de vida. (COFFITO, 2018)

A terapia ocupacional, no SAD do HGWA, atende aos cuidadores, como também capacita os pacientes a executarem as suas Atividades de Vida Diária, AVD com o auxílio de dispositivos de tecnologia assistiva.

As atividades da vida diária (AVD) são componente importante do papel de manutenção pessoal. O termo engloba todas as ações ligadas aos cuidados pessoais, e subdivide-se em Atividades Básicas de Vida Diária (AVBD) que compreende o alimentar-se, arrumar-se, vestirse, banhar-se, usar a toalete e a mobilidade. As Atividades Instrumentais de Vida Diária (AVDI) envolvem a verdadeira independência, pois compreende o controle das próprias medicações, alimento, proteção, finanças, e comunicações para segurança, negócios pessoais e sociabilidade. Estas requerem interação maior com os ambientes físicos e sociais se comparadas com a primeira subcategoria. (TROMBLY, 2005)

O terapeuta ocupacional (TO) é capacitado a ensinar o paciente a realizar as atividades que estejam fora de seu contexto funcional. A readequação da rotina, através de estratégias, 
neste caso, em particular, através da confecção de dispositivos de Tecnologia Assistiva capazes de resgatar a execução destas atividades, confecção esta, realizada, muitas vezes, junto ao cliente, após uma escuta e uma avaliação diferenciada.

"A tecnologia assistiva (TA) é um termo ainda muito novo que é utilizado para identificar todo o arsenal de recursos e serviços que contribuem para proporcionar ou ampliar habilidades funcionais de pessoas com deficiência, promovendo vida independente e inclusão." (COOK, H.1995 apud BRASIL, 2015).

Para que haja sucesso nas Estratégias Terapêuticas Ocupacionais aqui citadas é fundamental também a parceria com o cuidador. Este será orientado, junto ao paciente sobre as particularidades dos dispositivos, bem como sua conservação e o modo correto de utilizá-los, evitando assim, a desmotivação por parte do usuário, devido ao uso incorreto, bem como danos físicos, tais como as escaras decorrente do mau posicionamento das adaptações, por exemplo.

A TA tem como objetivo compensar as capacidades ausentes ou deficientes, permite que o indivíduo participe de tarefas de desempenho ocupacional, contribuindo para a qualidade de vida. (ANGELO; BUNING, 2005).

Ao citar o Modelo de Interação Pessoa-Ambiente, Angelo e Buning (2005), descrevem um processo dinâmico que ocorre entre o indivíduo e o ambiente, ou seja, quanto mais grave a deficiência, maior deve ser a modificação do ambiente para compensar a incapacidade criada pela disfunção.

Essa é a porta de entrada da TO do SAD durante o primeiro contato com o paciente, seus familiares e cuidadores. Através desta visão, atua acertivamente, na disfunção ocupacional do indivíduo, pois tem a capacidade de converter estes estados disfuncionais em estados de função ocupacional que acontecem através da análise de atividade. Esta é usada para identificar as propriedades inerentes a uma dada ocupação, tarefa ou atividade, tanto quanto as habilidades, proficiência e capacidades para completá-la.

Trombly (1995) fala claramente do efeito terapêutico da ocupação-como-fim, uma vez que, é de caráter intencional e têm seu significado individual, motivando assim a sua execução. Neste caso, temos a ocupação-como-fim, ressignificando a rotina do paciente tornando-o protagonista de sua vida e de suas escolhas.

Existe uma variedade de instrumentos utilizados pelo TO que facilitam a sua avaliação norteando-o acerca do desempenho do cliente, evidenciando as suas prioridades na execução das AVD, lazer e trabalho. No SAD do HGWA, é utilizado a Medida de Independência Funcional (MIF), já inserida no prontuário eletrônico da instituição.

"A MIF, emprega uma escala de sete pontos avaliando 18 itens em áreas de cuidados pessoais, controle dos esfincters, mobilidade, locomoção, comunicação e cognição social. Esta escala avalia o grau de incapacitação, tornando-se útil para averiguar as limitações, ou programar a avaliação inicial nos programas de reabilitação. Sua contagem, neste cenário é realizada de acordo com a observação direta ao paciente, durante as visitas domiciliares." (Universidade Federal do Paraná, 2003). Este Instrumento apresenta de boa a excelente confiabilidade, e tem sido objeto de longa validação.

Dessa forma, as estratégias terapêuticas Ocupacionais aqui utilizadas favorecem a execução das AVD, contribuindo para o enfrentamento das diversas enfermidades, fortalecendo os vínculos familiares e principalmente evidenciando o protagonismo do indivíduo em questão, mesmo estando em Cuidados Paliativos (CP). 


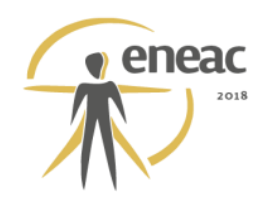

O Terapeuta Ocupacional é de fundamental importância, frente ao paciente em CP, pois, buscará possibilidades em aumentar a autonomia e as possibilidades do fazer, entendendo as atividades como possibilitadoras de experiências de potência, permitindo o resgate de capacidades remanescentes, bem como a criação de projetos a serem realizados. O principal objetivo é orientar o cuidador acerca dos estímulos positivos ao paciente e treiná-lo para que seja um facilitador da independência nas atividades de vida diária. (OTHERO, 2012).

Diante do exposto, objetivamos descrever as intervenções terapêuticas ocupacionais implementadas em um Serviço de Assistência Domiciliar e seus resultados considerando as Atividades de Vida Diária dos pacientes crônicos assistidos pelo referido trabalho.

\section{METODOLOGIA}

O estudo caracteriza-se como qualitativo, descritivo, do tipo relato de experiência. Fizemos uma referência da atuação do TO no SAD, e descrevemos casos de intervenções que potencializam a execução das AVD de pacientes acompanhados pelo referido profissional, e também os resultados dessas intervenções.

Os casos foram observados desde 2014, quando foi incluído o Serviço de Terapia Ocupacional à equipe multidisciplinar do SAD do Hospital Geral Dr. Waldemar Alcântara HGWA.

O SAD HGWA teve início em 2003, hoje, atende em média 210 pacientes, no município de Fortaleza-CE. O perfil dos pacientes com idade média de 68 anos, são de pessoas com doenças, cerebrovasculares, degenerativas, neuromusculares, pulmonares obstrutivas crônicas, encefalopatias e paliação.

Atualmente recebe pacientes de 7 hospitais de referência do Estado. As visitas domiciliares são realizadas por 5 equipes multidisciplinares composta por 6 médicos, 1 cirurgião, 6 enfermeiros, 9 fisioterapeutas, 3 assistentes sociais, 1 nutricionista, 1 psicólogo, 1 farmacêutico, 1 odontólogo, 1 técnica de saúde bucal, 1 fonoaudiólogo e 1 terapeuta ocupacional, 2 secretárias e 4 motoristas.

O referido estudo recebeu o consentimento dos pacientes e cuidadores envolvidos, através de termo de consentimento livre e esclarecido. Foram respeitados os aspectos éticos da pesquisa como preconizado resolução no 466, de dezembro de 2012.

\section{RESULTADOS}

\subsection{Atuação da terapia ocupacional na assistência domiciliar}

Os atendimentos Terapêuticos Ocupacionais acontecem de acordo com as demandas, identificadas na inclusão, durante a busca ativa e também as sinalizadas pela equipe multidisciplinar.

Após os devidos encaminhamentos, o terapeuta ocupacional, durante a primeira visita faz um levantamento do histórico ocupacional do paciente, avalia o ambiente domiciliar, tais como barreiras arquitetônicas, mobiliários, e aplica o instrumento que mensurará o nível de dependência do paciente, neste caso a MIF (Figura 1).

Durante a segunda visita são levantados os desejos do paciente e as possibilidades do ambiente domiciliar, e a partir desse momento têm-se início o processo de criação do dispositivo ali sugeridos e solicitado, sendo confeccionados os moldes e, durante as visitas futuras são apresentados os modelos dos dispositivos. 


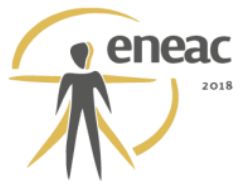

O processo educativo têm seu início antes mesmo da confecção do equipamento, pois o paciente será esclarecido acerca de suas capacidades, e mesmo em cuidados paliativos, o assistencialismo do cuidador deverá seguir em segundo plano sendo o usuário preparado para realizar as suas AVD.

Há a necessidade do emponderamento familiar e da capacitação do cuidador, pois este se tornará fonte primária de assistência nessa etapa, evitando frustações futuras, quando o dispositivo não estiver bem acoplado ou quando o paciente executar as atividades com baixa destreza, acarretando no não uso do equipamento ou em lesões (Brasil, 2015).

O cuidador é reconhecido como uma ocupação e integra a Classificação Brasileira de Ocupações (CBO), que define o cuidador como alguém que "cuida a partir dos objetivos estabelecidos por instituições especializadas ou responsáveis diretos, zelando pelo bemestar, saúde, alimentação, higiene pessoal, educação, cultura, recreação e lazer da pessoa assistida". É a pessoa, da família ou da comunidade, que presta cuidados à outra pessoa de qualquer idade, que esteja necessitando de atenção por estar acamada, com limitações físicas ou mentais, com ou sem remuneração (BRASIL, 2008).

Este é um dos motivos da necessidade de amparar o cuidador, através de escutas diferenciadas, de encontros mensais que tratem o seu bem estar e a sua saúde, bem como capacitá-lo evitando frustações e desentendimentos. Dessa forma o cuidador é tão parceiro quanto o usuário em questão, pois sem o seu devido apoio, essas ações seriam mais laboriosas de acontecer.

Dessa forma as sugestões de dispositivos são apresentados aos pacientes e cuidadores, e após aprovados, são criados os moldes, tendo início a sua confecção. Geralmente iniciamos a confecção nas dependências do SAD, devido ao curto tempo para as visitas domiciliares e após agendamento com o paciente, finalizamos a sua confecção in loco (Foto 1), escutando sempre as sugestões do paciente em questão (escuta individualizada).

Tabela 1 - Instrumento para Medida de Independência Funcional

\begin{tabular}{|c|c|c|c|c|}
\hline \multirow[b]{2}{*}{$\begin{array}{l}N \\
i \\
V \\
E \\
1 \\
S\end{array}$} & \multicolumn{2}{|c|}{$\begin{array}{l}\text { Inde pendente } \\
7 \text { - Independência completa (Com segurança e tempo nomal) } \\
6 \text { - Independência modificada (Ajuda técnica) }\end{array}$} & \multicolumn{2}{|c|}{ SEM ASSISTÉNCIA } \\
\hline & \multicolumn{2}{|l|}{ 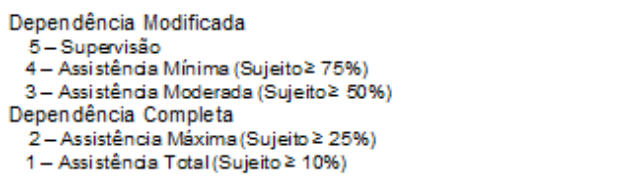 } & \multicolumn{2}{|c|}{ COM ASSISTÉNCIA } \\
\hline Avaliação & \multicolumn{2}{|l|}{ Atividades } & $2^{\circ} \mathrm{Av}$. & $3^{\circ} \mathrm{Av}$. \\
\hline & Autocuidado & 11 & 11 & 11 \\
\hline A. & Alimentaçäo & & & \\
\hline B. & Higiene pess osl: apresentação e aparêndas. & & & \\
\hline c. & Banho: laver o corpo & & & \\
\hline D. & Vestir: metade superior do como & & & \\
\hline E. & Vestir: metade inferior do corpo & & & \\
\hline \multirow[t]{2}{*}{$\mathrm{F}$. } & Utilizacăo do vaso sanitário & & & \\
\hline & \multicolumn{2}{|l|}{ Controle dos esfincteres } & & \\
\hline G. & Controle da urina: frequêncis de incontinêncis & & & \\
\hline \multirow[t]{2}{*}{ H. } & Controle das fezes & & & \\
\hline & \multicolumn{2}{|l|}{ Mobilidade } & & \\
\hline I. & Transferências: leito, cadeira, cadeira de rodas & & & \\
\hline J. & Transferências: vaso sanitánio & & & \\
\hline \multirow[t]{2}{*}{ K. } & Transferências: banheira ou chuveiro & & & \\
\hline & \multicolumn{2}{|l|}{ Locomocãa } & & \\
\hline \multirow[t]{2}{*}{ L. } & \multirow{2}{*}{ Marcha/Cadeira de rodas } & $\mathrm{M}$ & \begin{tabular}{|l|}
$\mathrm{M}$ \\
\end{tabular} & $\mathrm{M}$ \\
\hline & & $\mathrm{CR}$ & $\mathrm{CR}$ & $\mathrm{CR}$ \\
\hline M. & \multicolumn{4}{|l|}{ Comunicação } \\
\hline \multirow{2}{*}{ N. } & \multirow{2}{*}{ Compreensão } & A & A & $\mathrm{A}$ \\
\hline & & $\mathrm{VI}$ & \begin{tabular}{|ll}
$\mathrm{VI}$ \\
\end{tabular} & $\mathrm{Vl}$ \\
\hline \multirow{3}{*}{0.} & \multirow{2}{*}{ Expressão } & $\mathrm{V}$ & $\mathrm{V}$ & $\mathrm{V}$ \\
\hline & & NV & NV & NV \\
\hline & Conhecimento Social & & & \\
\hline P. & Interacão Social & & & \\
\hline Q. & Resoluçăo de Problemas & & & \\
\hline R. & Memória & & & \\
\hline Total & & & & \\
\hline
\end{tabular}

Fonte: UFPR - Terapia Ocupacional Aplicada a Neurologia - 2003 


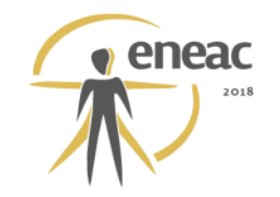

Foto 1 - Confecção In loco

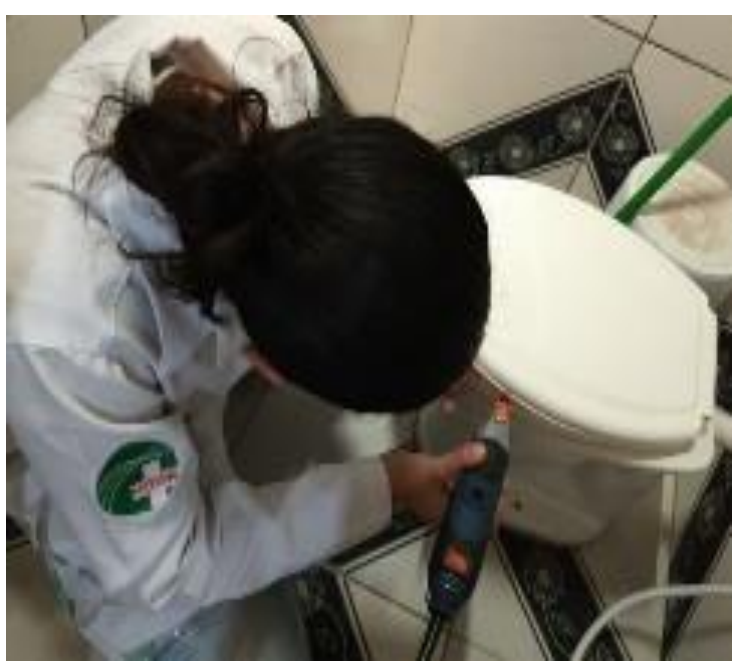

Fonte: Arquivo pessoal da autora 1 - 2017

\subsection{Descrição de casos}

Caso 1 - Nome: MJ, 55 anos, portadora de Esclerose Lateral Amiotrófica há 8 anos. Tem como demanda principal a utilização do computador, sentada à $90^{\circ}$ à mesa do dispositivo. Apresenta movimentação ativa preservada do dedo indicador esquerdo, rotação assistida da cabeça e disartria importante. O escore total da FIM somou 47, sendo 13 para domínio motor o que indica total dependência e 34 para domínio cognitivo, resultando em independência modificada. As Estratégias Terapêuticas Ocupacionais utilizadas foram uma adaptação para mouse (Foto 2), a confecção do Dispositivo Antigravitacional para a Cabeça (Foto 3). Os resultados alcançados foram a saída do leito durante o dia, com a plena utilização do computador sentada à 90 (Foto 4), a paciente referiu também uma diminuição do peso da cabeça sob a coluna cervical, resultando em maior conforto na posição sentada.

\section{Foto 2 - Adaptação para mouse}

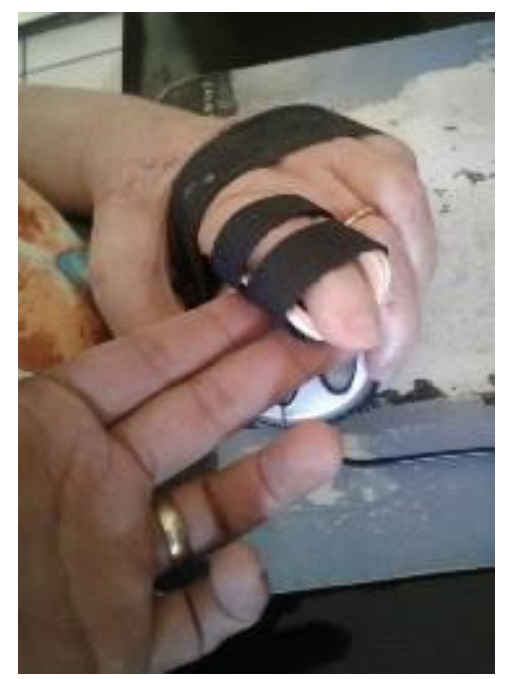

Fonte: Arquivo pessoal da autora $1-2015$ 


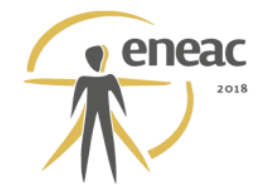

Foto 3 - Dispositivo Antigravitacional da cabeça

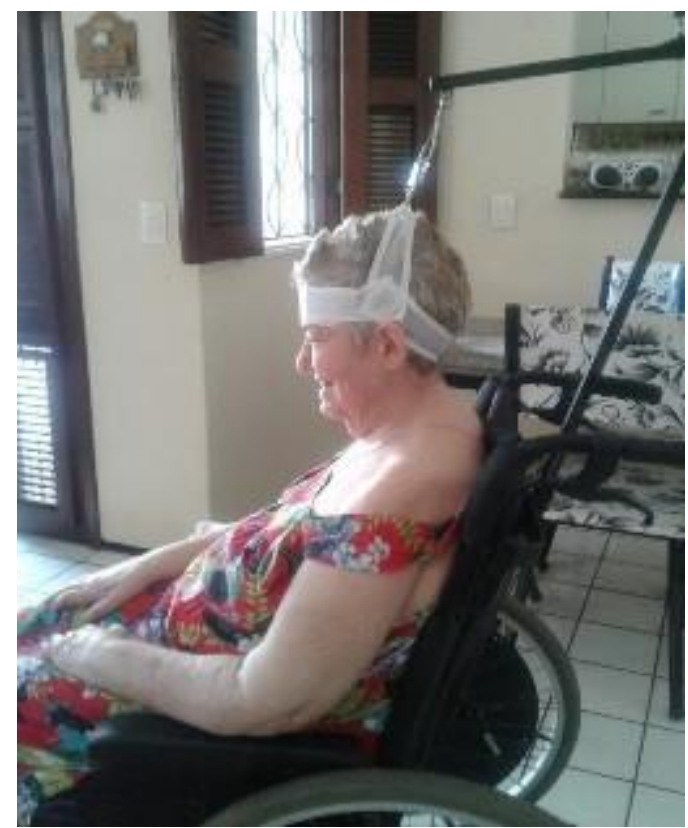

Fonte: Arquivo pessoal da autora 1 - 2017

Foto 4 - Utilização do computador sentada à $90^{\circ}$

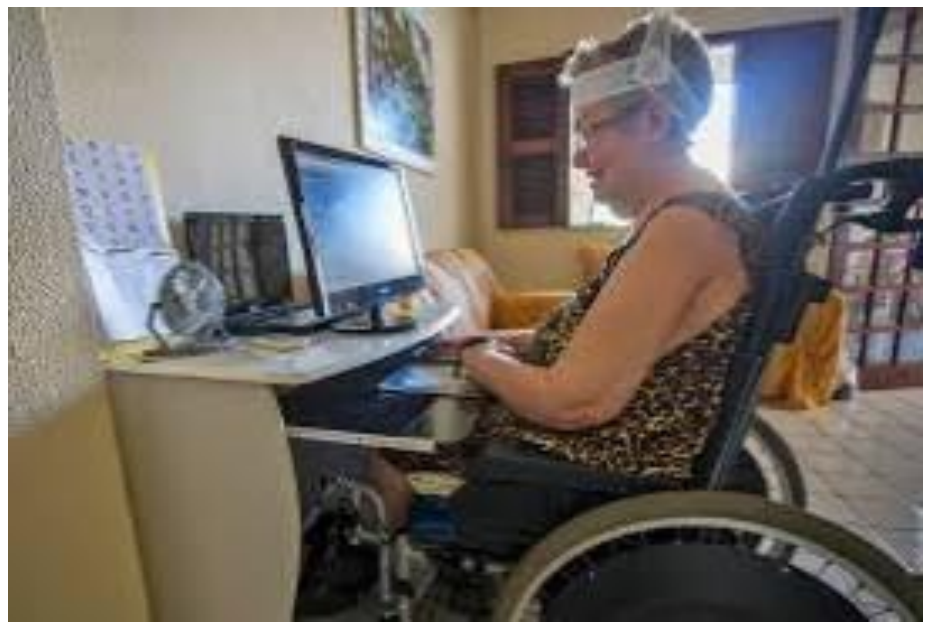

Fonte: Arquivo pessoal da autora $1-2017$

Caso 2. EF, 47 anos, Portador de Esclerose Lateral Amiotrófica, apresenta dificuldade e fadiga muscular ao alimentar-se. Apresentou, no momento da avaliação, FIM total igual a 78, sendo 44 para resultado motor o que define a dependência modificada e 34 para resultado cognitivo, podendo ser considerado independência total para esta categoria. A Estratégia Terapêutica Ocupacional utilizada foi o engrossador de preensão palmar confeccionado com material de baixo custo, látex e arame galvanizado (Foto 5). Resultados alcançados: Diminuição do cansaço durante as refeições mais demoradas. Paciente referiu mais qualidade de vida. 


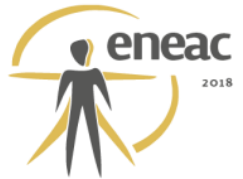

Foto 5, Engrossador de preensão palmar

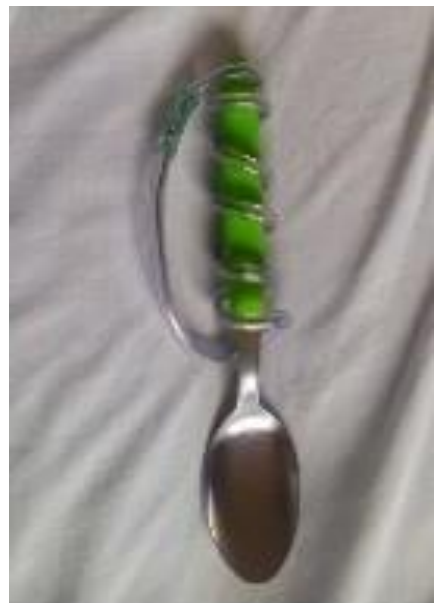

Fonte: Arquivo pessoal da autora $1-2017$

Caso 3. CG, 18 anos, sequela de Trauma Raquimedular por projetil de arma de fogo, apresentou contraturas em MMSS, resultando em deformidade. No momento da avaliação o cálculo total FIM somou em 48, sendo parcial motor igual a 13 e parcial para cognição igual a 35. O Paciente apresenta total dependência motora com cognitivo preservado. A Estratégia Terapêutica Ocupacional utilizada foi a confecção de órtese de posicionamento funcional in loco, com material de baixo custo (Foto 6), apresentando como resultados o resgate da posição funcional dos membros superiores, prevenção de contraturas e deformidades.

\section{Foto 6 - Órtese confeccionada com material de baixo custo}

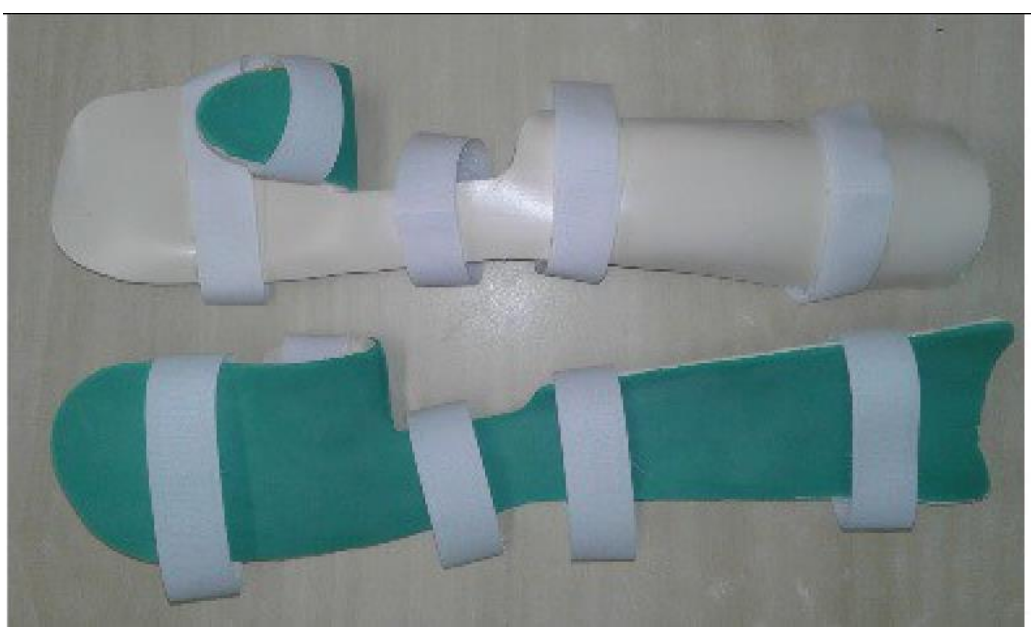

Fonte: Arquivo pessoal da autora 1 - 2018

\section{DISCUSSÃO}

A desospitalização é um dos aspectos da $A D$ que contribuem para o processo de alta hospitalar, prevenindo as intercorrências clínicas, a partir do acompanhamento regular da equipe multidisciplinar; diminuindo os riscos de infecções hospitalares devido ao longo tempo 


\section{$\dot{\pi}^{\text {eneac }}$}

de permanência, dando aporte emocional ao paciente e cuidador, potencializando a autonomia do paciente no cuidado fora do hospital (BRASIL, 2012).

O resgate da autonomia do paciente e a diminuição da sobrecarga de trabalho do cuidador é um dos papeis do terapeuta ocupacional, uma vez que, é o profissional responsável pela independência funcional do indivíduo.

No caso 1, a implementação do dispositivo antigravitacional para cabeça gerou impacto na mobilidade e transferência do paciente, contribuiu para seu posicionamento funcional e conforto, favorecendo o uso do computador, este último foi complementado pela órtese de adaptação para o uso do mouse. Caso contrário a paciente ficaria impossibilitada de sentarse a $90^{\circ}$ conforme seu desejo inicial.

O primeiro dispositivo poderia contribuir também para outras patologias que impossibilitam a sustentação ativa da cabeça, pois durante a reabilitação, sempre almejamos o máximo de desenvoltura do paciente, não esquecendo a qualidade, para tanto, em alguns momentos são imprescindíveis os dispositivos de tecnologia assistiva.

Segundo a International Standards Organization - ISO órteses são dispositivos aplicado externamente ao corpo humano para modificar as características funcionais ou estruturais do sistema musculoesquelético; esse equipamento pode ser confeccionado de diversos materiais e auxiliam no processo de estabilização e/ou recuperação de processos de lesões, envelhecimento, processos congênitos, entre outros.

O caso 2 utilizamos o engrossador universal para preensão palmar, que auxiliou na conservação de energia durante as refeições quando o paciente realiza a ação de levar o talher a boca resultando em uma alimentação independente sem a intervenção do cuidador, resultando em uma melhor qualidade de vida.

O problema da alimentação, segundo Trombly (2005) é a incapacidade de preensão e/ou levar a mão a boca, sendo sugerido a utilização de um engrossador, sempre levando em consideração as particularidades do paciente.

No caso 3, utilizamos uma órtese estática, cuja função é manter o correto alinhamento articular, proporcionando apoio, estabilização, proteção ou imobilização, evitando deformidades, pois impedem o desenvolvimento de contraturas pelo inadequado posicionamento. Devido à escassez de recursos financeiros da instituição optou-se pela utilização do policloreto de polivinila - PVC.

A OMS em 1986 publicou os princípios que regem a atuação da equipe multiprofissional referenciando a qualidade de vida em Cuidados Paliativos. Estes princípios foram reafirmados na sua revisão em 2002 e o $5^{\circ}$ princípio fala claramente em "Oferecer um sistema de suporte que possibilite o paciente viver tão ativamente quanto possível, até o momento da sua morte". Referenciando a qualidade de vida e bem-estar que englobam diversos segmentos da vida. Enfatiza também que o Viver ativamente, e não simplesmente viver, nos remete à questão da sobrevida "a qualquer custo", que esperamos combater (MATSUMOTO, 2012).

\section{CONCLUSÃO}

As estratégias terapêuticas ocupacionais aqui citadas são a prova de que, mesmo o paciente apresentando comprometimento motor severo comprovado através da Medida de Independência Funcional, é capaz de ser o protagonista de sua vida, é capaz de fazer as suas escolhas e de fazer a sua práxis evidente, e mesmo em cuidados paliativos ele poderá executar suas atividades de vida diária quando bem equipado e bem orientado.

Este estudo é um incentivo para uma escuta diferenciada, tanto do paciente, quanto do cuidador, permitindo a vida fluir com o mínimo de sofrimento possível, frente a tantas dores e 
incertezas, comprovando que mesmo em Assistência Domiciliar, mesmo em cuidados paliativos é possível e necessário investir nesses pacientes.

\section{REFERÊNCIAS BIBLIOGRÁFICAS}

ANGELO. Jennifer; BUNING. Mary Ellen. Adaptação de Alta Tecnologia para Compensar a Deficiência. In: TROMBLY, C. A.; RADOMSKI, M. V. Terapia ocupacional para disfunções físicas. 5. ed. São Paulo: Livraria Santos Editora Ltda, 2005.

Brasil. Ministério da Saúde. Diretrizes de Atenção à Reabilitação da Pessoa com Traumatismo Cranioencefálico. Brasília: Ministério da Saúde, 2015.

Brasil. Ministério da Saúde. Melhor em Casa: A segurança do Hospital no Conforto do seu Lar. Brasília: Ministério da Saúde, v. 1, 2012.

Brasil. Ministério da Saúde. Melhor em Casa: A segurança do Hospital no Conforto do seu Lar. Brasília: Ministério da Saúde, v. 2, 2013.

Brasil. Ministério da Saúde. Guia Prático do Cuidador. Série A. Normas e Manuais Técnicos.Brasília: Ministério da Saúde, 2008.

COFFITO, Definição da Terapia Ocupacional. [2018]. Disponível em:

$<$ https://www.coffito.gov.br/nsite/?page id=3382>. Acesso em:13/02/2018.

EGGERS. O. Terapia Ocupacional no Tratamento da hemiplegia do adulto. Tradução de

J. Israel Lemos, 1aㅡ edição. Rio de Janeiro: Colina, 1984.

MATSUMOTO, D.Y. Manual de Cuidados Paliativos ANCP. Ampliado e atualizado $2^{\underline{a}}$ edição. Organizadores: Ricardo Tavares de Carvalho Henrique Afonseca Parsons. Academia Nacional de Cuidados Paliativos, 2012.

OTHERO, B.M. Manual de Cuidados Paliativos ANCP. Ampliado e atualizado $2^{\mathrm{a}}$ edição. Organizadores: Ricardo Tavares de Carvalho Henrique Afonseca Parsons. Academia Nacional de Cuidados Paliativos, 2012.

TROMBLY, C.A. Restauração do Papel de Pessoa Independente. In: TROMBLY, C. A.; RADOMSKI, M. V. Terapia ocupacional para disfunções físicas. 5. ed. São Paulo: Livraria Santos Editora Ltda, 2005.

, C.A. Ocupação. In: TROMBLY, C. A.; RADOMSKI, M. V. Terapia ocupacional para disfunções físicas. 5. ed. São Paulo: Livraria Santos Editora Ltda, 2005.

UNIVERSIDADE FEDERAL DO PARANÁ. Terapia Ocupacional Aplicada a Neurologia. [2003]. Disponível em: <https://toneurologiaufpr.wordpress.com/2013/04/01/medida-de- independenciafuncional-mif/>. Acesso em:13/02/2018. 